\title{
Automatic Face Segmentation Based on the Level Set Method
}

\author{
Chaoyi Zhang \\ School of Computer Science, \\ Northwestern Polytechnical University \\ Shaanxi Key Laboratory of Speech and \\ Image Information Processing \\ Xi'an, China \\ chaoyi_zhang@126.com
}

\author{
Yanning Zhang \\ School of Computer Science, \\ Northwestern Polytechnical University \\ Shaanxi Key Laboratory of Speech and \\ Image Information Processing \\ Xi'an, China \\ ynzhang@nwpu.edu.cn
}

\author{
Zenggang Lin \\ School of Computer Science, \\ Northwestern Polytechnical University \\ Shaanxi Key Laboratory of Speech and \\ Image Information Processing \\ Xi'an, China \\ talcool@163.com
}

\begin{abstract}
Face segmentation is a crucial problem in face recognition and other areas like pedestrian detection. Level set methods have been widely used in image segmentation. In this paper, the modified level set evolution equation is used in the level set method to segment the face. Based on the signed pressure force (SPF) function, a variable parameter signed pressure force (VPSPF) function is proposed. The proposed function avoids the disadvantage of the SPF function because it does not need the image intensity to satisfy specific condition. Our method combines the GAC model and the $\mathrm{C}-\mathrm{V}$ model. Let the VPSPF function which contains the statistical information inside and outside the contour substitute the edge stopping function (ESF) in the GAC model. It not only has the advantages which the Selective Binary and Gaussian Filtering Regularized Level Set (SBGFRLS) method demonstrates but also can solve the problem the SBGFRLS method cannot handle. Experiment on the synthetic image using our method shows better performances than using the SBGFRLS method. Experiment results in the images from the ORL Database of Faces and the general images which have more than one faces show our method's validity and superiority.
\end{abstract}

Keywords- face segmentation; level set method; signed pressure force function; GAC model; $C-V$ model

\section{INTRODUCTION}

Image segmentation is the crucial problem in image analysis and image understanding. The face segmentation is a significant initial step for face recognition. It is also an important problem of computer vision and pattern recognition for the reason that it widely used not only in face recognition but also in other areas like pedestrian detection. The active contour model (ACM) is one of the most successful techniques in image segmentation. The fundamental idea of ACM is to evolve a curve according to some certain constrains to extract the desired object. Geometric active contour model is one type of active contour model and it is widely used in segmentation.

Geometric active contours are based on the theory of curve evolution and the level set method. They are represented implicitly as level sets of two dimensional distance functions which evolve according to an Eulerian formulation and are hailed as the solution to the problem of required topological changes during curve evolution [1]. The existing geometric active contour models are categorized into edge-based models and region-based models. The geodesic active contour (GAC) model is one of the most famous edge-based models [2]. It

Supported by the Basic Research Foundation of Northwestern Polytechnical University (JC201122) utilizes image gradient to construct the ESF to stop the contour evolution on the object boundaries. In order to increase the efficiency of the convergence to boundary concavities, Caselles et al. added a "convergent force" to the GAC model to increase the propagation speed. This "convergent force" can not only fast the convergence speed at smoothing areas, but also make the curve shrink in spite of the curvature is negative. However, the GAC model cannot detect the object when the initial contour does not surround its boundary. One of the most popular region-based models is the $\mathrm{C}-\mathrm{V}$ model [3]. It is based on Mumford-Shah segmentation techniques and has been applied to binary phase segmentation. Unlike the classic active contour based on the gradient of a given image, the $\mathrm{C}-\mathrm{V}$ model can detect the edges whether there are clear boundaries in the image or not. There is another advantage. The global information is considered in the $\mathrm{C}-\mathrm{V}$ model and so the model has the global optimization ability. Just one initial closed contour can detect all the edges of the object with internal holes. Modifications and improvements have been proposed to change the behavior in a variety of applications. A novel region-based active contour model is implemented with SBGFRLS method [4]. This method shows advantages over traditional level set method in terms of both efficiency and accuracy.

This paper is organized as follows. In Section II, we improved the SPF function to VPSPF function and the SBGFRLS method is also modified based on the improved function. We use synthetic image to show the advantage of our method and apply our method to face segmentation. Experiments results are shown in Section III. We do the experiments on the images from the ORL Database of Faces and on the general real images. In Section IV we draw the conclusion.

\section{THE IMPROVED SBGFRLS METHOD AND ITS APPLICATION TO FACE SEGMENTATION}

The SBGFRLS method is proposed in [4]. The advantages of this method are as follows. First, the contours can be stopped at the weak or blurred edges by the SPF function. Second, despite the position of the initial contour, both the interior and exterior boundaries can be automatically detected. Third, the ACM with the SBGFRLS method can segment not only the desired object but also the other objects. Forth, a binary 
function is used to initialize the level set function. It is more efficient to construct than the widely used signed distance function. Finally, the simple finite difference scheme can implement the proposed algorithm efficiently. Though it has many advantages, there is one main drawback: if there is not only one object in the image, the intensities of the objects and the background must satisfy a specific requirement. In this section, we modified the SBGFRLS method through improving the SPF function to VPSPF function. Our method can be used more widely because the requirement does not need to be satisfied. Then we apply our method to face segmentation.

\section{A. The VPSPF function}

In [5], the SPF function which is derived from the image has values in the range $[-1,1]$ and the value is smaller on the region of interest. It modulates the sign of the SPF function using region information so that the contour expands when it is inside the object and shrinks when it is outside the object. This helps to segment the object which has weak edges. In [4], the SPF function is constructed as follows:

$$
\operatorname{spf}(I(x, y))=\frac{I(x, y)-\frac{c_{1}+c_{2}}{2}}{\max \left(\left|I(x, y)-\frac{c_{1}+c_{2}}{2}\right|\right)}
$$

where $c_{1}$ and $c_{2}$ are the average intensities inside and outside the contour respectively.

The signs of the SPF function inside and outside the object are opposite and it is decided by the value of intensities inside and outside the object. The corresponding level set formulation of the GAC model using the PDE with the embedding function is as follows:

$$
\frac{\partial \phi}{\partial t}=g|\nabla \phi|\left(\alpha+\operatorname{div}\left(\frac{\nabla \phi}{|\nabla \phi|}\right)\right)+\nabla g \cdot \nabla \phi
$$

Substituting the SPF function represented as (1) for the ESF in (2), the formulation of the curve evolution can be written as follows:

$$
\frac{\partial \phi}{\partial t}=\operatorname{spf}(I(x, y))|\nabla \phi|\left(\alpha+\operatorname{div}\left(\frac{\nabla \phi}{|\nabla \phi|}\right)\right)+\nabla \operatorname{spf}(I(x, y)) \cdot \nabla \phi
$$

The above SPF function and the curve evolution equation are based on the image which has only one object. When there is more than one object in the image, as shown in Fig. 1, it is assumed there are $N$ objects in the image. The object's area is represented as $S_{i}$ and its intensity is represented as $I_{i}$. The initial contour is outside all the objects. The background's intensity is $I_{b}$ and its area inside and outside the contour are $S_{b i}$ and $S_{b o}$ respectively. It is assumed that the value of the background intensity is either the highest or the lowest. However, similar to the $\mathrm{C}-\mathrm{V}$ model [6], this model cannot handle the case when the background intensity is neither the highest nor the lowest. Additionally, it is assumed that the background intensity is the lowest and $I_{b} \leq I_{1} \leq I_{2} \leq \cdots \leq I_{N}$ in order to analysis conveniently. $c_{1}$ and $c_{2}$ are the average intensities and in the multiobjects condition they can be represented as:

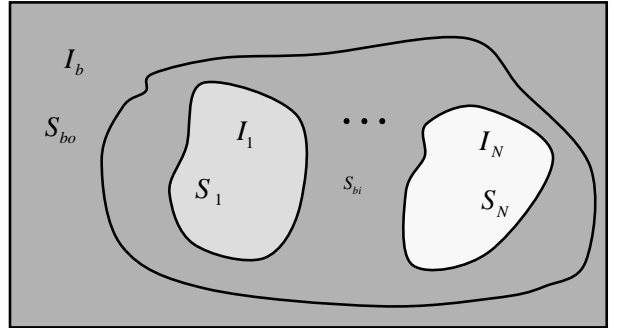

Figure 1. Demonstration of segmentation for multi-objects with different intensities.

$$
\left\{\begin{array}{l}
c_{1}=\left(\sum_{k=1}^{N} I_{k} S_{k}+I_{b} S_{b i}\right) /\left(\sum_{k=1}^{N} S_{k}+S_{b i}\right) \\
C_{2}=I_{b}
\end{array}\right.
$$

So the SPF function can be simplification as:

$$
\operatorname{spf}(I(x, y))=\frac{\left(I(x, y)-I_{b}\right) S_{b i}+\sum_{k=1}^{N}\left(I(x, y)-\frac{I_{b}+I_{k}}{2}\right) S_{k}}{\sum_{k=1}^{N} S_{k}+S_{b i}}
$$

The denominator of (1) is omitted because the denominator is a constant for a specific image and it will not affect the sign of the SPF function. When the pixel is in the background, the sign of the SPF function is negative. If $I_{1}>\left(I_{b}+I_{N}\right) / 2$, it is certain that the sign of the SPF function is positive for all pixels in any objects. In this condition, this method works. Otherwise, the conclusion about the sign of the SPF function is not always true. The SBGFRLS method may fail to detect all the edges of all the objects.

We improved the SPF function by adding a parameter $\beta$ to the original SPF function, then the improved SPF function which we call variable parameter signed pressure force (VPSPF) function is constructed as follows:

$$
v p s p f(I(x, y))=\frac{I(x, y)-\beta \cdot \frac{c_{1}+c_{2}}{2}}{\max \left(\left|I(x, y)-\frac{c_{1}+c_{2}}{2}\right|\right)}
$$

Substituting the VPSPF function for SPF function in (3), we get the improved formulation of the curve evolution:

$$
\frac{\partial \phi}{\partial t}=\operatorname{vpspf}(I(x, y))|\nabla \phi|\left(\alpha+\operatorname{div}\left(\frac{\nabla \phi}{|\nabla \phi|}\right)\right)+\nabla \operatorname{vpspf}(I(x, y)) \cdot \nabla \phi
$$

In the condition of Fig. 1, the VPSPF function is as follows:

$$
\operatorname{vpspf}(I(x, y))=I(x, y)-\beta \cdot \frac{\left(\sum_{k=1}^{N} I_{k} S_{k}+I_{b} S_{b i}\right) /\left(\sum_{k=1}^{N} S_{k}+S_{b i}\right)+I_{b}}{2}
$$


When the pixel is in the background, the VPSPF function can be written as:

$$
\operatorname{vppp}(I(x, y))=\frac{(1-\beta) I_{b} S_{b i}+\sum_{k=1}^{N} \frac{(2-\beta) I_{b}-\beta I_{k}}{2} S_{k}}{\sum_{k=1}^{N} S_{k}+S_{b i}}
$$

When the pixel is in Object $i$, the VPSPF function is:

$$
\operatorname{vpspf}(I(x, y))=\frac{\left(I_{i}-\beta I_{b}\right) S_{b i}+\sum_{k=1}^{N}\left(I_{i}-\frac{\beta\left(I_{b}+I_{k}\right)}{2}\right) S_{k}}{\sum_{k=1}^{N} S_{k}+S_{b i}}
$$

Let the VPSPF function expressed as (9) be negative and the VPSPF function expressed as (10) be positive, we can easily get that:

$$
\frac{2 I_{b}}{B}<\beta<\frac{2 I_{N}}{B}, B=\left(\sum_{k=1}^{N} I_{k} S_{k}+I_{b} S_{b i}\right) /\left(\sum_{k=1}^{N} S_{k}+S_{b i}\right)+I_{b}
$$

When $\beta$ satisfies this inequality, the sign of VPSPF function in (9) is negative and the sign for all the pixels in all the objects is positive. So this VPSPF function can handle the problems which SPF function may failed to solve.

We use the simple synthetic image to illustrate the advantage of our model. There are three rectangles in the image from the left to the right. In Fig. 2, the first image is the original image and the second is the image with the initial contour. The third image is the result which uses the SPF function and the last image is the result which uses our VPSPF function.

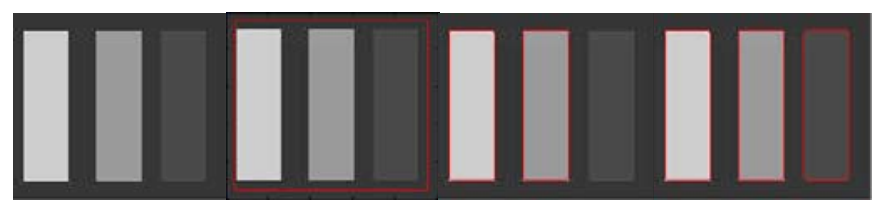

Figure 2. Comparisons of the SBGFRLS method using SPF function and our method using VPSPF function. The first image is the original image, the second imge shows the initial contour, the third image is the result of the SBGFRLS method and the last is the result of ours.

In the synthetic image, $I_{b}=55, I_{1}=75, I_{2}=155, I_{3}=205$, it does not satisfy the inequality $I_{1}>\left(I_{b}+I_{N}\right) / 2$ so the original method fails. The three rectangles are not all segmented and only the two rectangles which have higher intensities are segmented. The image of our result is shown and all the three rectangles are precisely segmented. Experiments on the synthetic image show our method is better than the original method. In this experiment, we chose $\beta=0.72, \alpha=10, \Delta t=1$. Using the algorithm of numerical solution of PDE and after about 110 iterations, we get the final segmentation result.

\section{B. Implementation based on VPSPF function}

In traditional level set methods [1, 2, 7-9], the level set function $\phi$ can develop shocks, very sharp or flat shape during the evolution. Because of this, the further computation is inaccurate. In order to avoid these problems, a common numerical method is to initialize the function $\phi$ as a signed distance function before the evolution. During the evolution, the function $\phi$ needs to be re-initialized so that it can maintain as a signed distance function. The standard re-initialization method is to solve the equation below:

$$
\frac{\partial \phi}{\partial t}=\operatorname{sign}\left(\phi_{0}\right)(1-|\nabla \phi|)
$$

However, if $\phi_{0}$ is not smooth or $\phi_{0}$ is much steeper on one side of the interface than the other, the zero level set moves away from the interface. Furthermore, when the level set function is far away from a signed distance function, these methods may not be able to re-initialize the level set function to a signed distance function. In addition, it is difficult to decide when and how to do the re-initialization which is a very expensive operation. In order to solve these problems discussed above, a novel level set method SBGFRLS method is proposed in [4].

According to [4], the method is very easy to implement in practice. The curvature-based term $\operatorname{div}(\nabla \phi /|\nabla \phi|)|\nabla \phi|$ can be substituted by the Gaussian filtering process because the Gaussian filtering process is used to further regularize the level set function. Moreover, for the reason that the VPSPF function utilizes the statistical information of regions and it has a larger capture range and capacity of leakage, the term $\nabla v p s p f(I(x, y)) \cdot \nabla \phi$ in (7) can also be removed [10]. Finally, the simple level set formulation can be written as follows:

$$
\frac{\partial \phi}{\partial t}=\operatorname{vpspf}(I(x, y)) \cdot \alpha|\nabla \phi|
$$

\section{Apply the method to face segmentation}

According to the method discussed above, the level set method represented in (13) can handle the following cases well: (1) There is more than one object in the image. (2) The object average intensity and the background average intensity are not homogenous; it is not need to satisfy that the intensities in the object are the same. (3) There are noises in the image. But, when the object average intensity and the background average intensity are homogenous this method may failed. Also, when the value of the pixels near the object's edge is closer to the value of the background intensities, the segmented result will not include these pixels. It is worth nothing that the traditional model like the $\mathrm{C}-\mathrm{V}$ model may also fail in these cases.

The face images, including the images in the ORL Database of Faces and the general real images, are not all satisfy the condition made above. So, when we apply this method to face segmentation in these images, the preprocessing step should be taken. In general, the value of face intensity is often higher than other pixel values. So, before using the level set method we increase the contrast of the image. It is very helpful to the segmentation because the difference between the object and the background is enlarged. Because the method may segment other areas which are not faces in the image of ORL Database of Faces and noted that the face area is the biggest one, we delete the smaller areas so that only the face is preserved. The experimental results are shown in Section III. 


\section{EXPERIMENTAL RESULTS}

The experimental results are shown in this section. We apply the method to face segmentation. Both the images in ORL Database of Faces and the general real images are used to be segmented. Our experiments are implemented in Matlab 7.8 on a $3.2 \mathrm{GHz}$ Intel Pentium 4 PC.

We apply our model to face segmentation. The face images in the ORL Database of Faces are used to be segmented. The first row in Fig. 3 shows the original image and the results of the SBGFRLS method proposed in [4]. The second row shows the results using our method and the last three is the results after the post-processing.

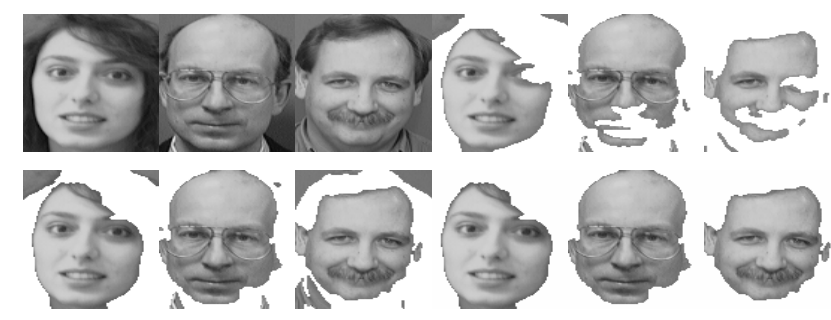

Figure 3. Comparisons of the SBGFRLS method and our method in face segmentation. The first row: the original images and the results of SBGFRLS method. The second row: the original results of our method and the results after the post-processing.

We set $\beta_{1}=0.87, \beta_{2}=0.97, \beta_{3}=0.94$ respectively in the above three images. Our model can segment more than one object so the background whose intensity is more close to the cheek is segmented, like the areas on the top of the image. For this problems, noted that among all the segmented areas the face area is the biggest one, so we can do the post-processing to delete the unrelated areas which are all smaller than the face areas. We compute the number of the pixels who are connected in the image and only preserve the biggest area. The final result is shown in the last row from which we can see that the segmented region includes nearly all the main parts of the face except few ears are missed. From Fig. 3, we can see that our method can get better results than the SBGFRLS method. Also, we only add one parameter in the original SPF function and it is only a coefficient, so the computational complexity does not increase much.

More results are shown in Fig. 4. From the result we can see that the faces are all segmented presicesly and completely.

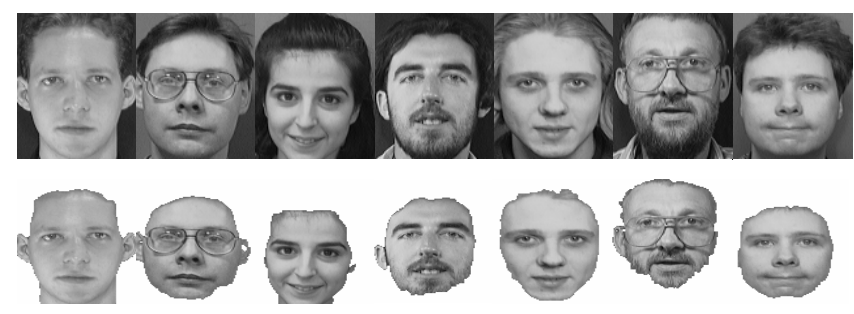

Figure 4. The results of face segmentation in the images from the ORL Database of Faces. The first row shows the origianl image and the second row shows the result.

Finally, we apply our model into the real image. The results are shown in Fig.5. From the result we can see that the area under the face whose intensity is more close to the face is also included in the segmented result. Though it cannot be avoided, it is worth nothing that from the result we can also know the position of the face and how many faces are in the image. In addition, because there is more than one face in the image, we set a threshold to delete the unrelated areas instead of conserving the biggest one. If the threshold is set bigger, the incomplete face will be removed and otherwise it will be remained in the result as shown in Fig. 5.
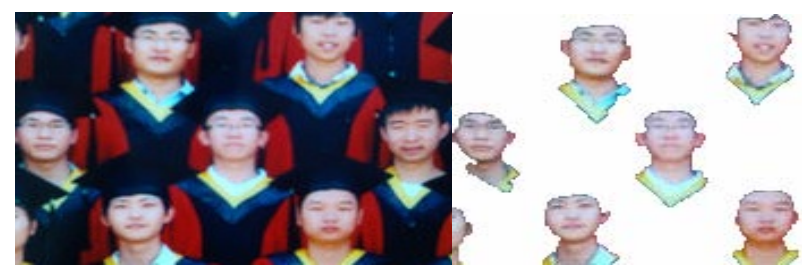

Figure 5. The result of segmentation in real image. The left one is the original image and the right one is the segmented image.

\section{CONCLUSION}

In this paper, we proposed the VPSPF function which improves the SPF function and according to the VPSPF function modified the equation of level set evolution. It has the advantages which the SBGFRLS method demonstrates, like the effectiveness and efficiency. Moreover, the proposed method avoids the drawbacks of the SBGFRLS method that the images intensities should not meet a specific requirement. Experiment on synthetic image proves the advantage of our method. Experiments on the face images show that our method can not only segment the images in the database but also segment the general images which have more than one face. This shows that our method is effective and robust in face segmentation.

\section{ACKNOWLEDGMENT}

This work is supported by the Basic Research Foundation of Northwestern Polytechnical University (JC201122).

\section{REFERENCES}

[1] R. Malladi, J. A. Sethian, and B. C. Vemuri. Shape modeling with front propagation: a level set approach. IEEE Transactions on Pattern Analysis and Machine Intelligence, 17(2):158-175, 1995

[2] V. Caselles, R. Kimmel, G. Sapiro, Geodesic active contous, International Journal of Computer Vision 22 (1):61-79, 1997

[3] T. Chan, L. Vese, Active contours without edges, IEEE Transactions on Image Processing 10 (2):266-277, 2001

[4] Kaihua Zhang, Lei Zhang, Huihui Song, et al, Active contours with selective local or global segmentation: A new formulation and level set method, Image and Vision Computing, 28 (2010) 668-676

[5] Chenyang Xu, Anthony Yezzi Jr., Jerry L. Prince, On the relationship between parametric and geometric active contours, Proc. Of $34^{\text {th }}$ Asilomar Conference on Signals, Systems, and Computers, pp. 483-489, October 2000

[6] L.A. Vese, T.F. Chan, A multiphase level set framework for image segmentation using the Mumford-Shah model, International Journal of Computer Vision 50:271-293, 2002

[7] S. Osher, R. Fedkiw, Level set methods and dynamic implicit surfaces, Spinger-Verlag, New York, 2002.

[8] J. A. Seyhian, Level set methods and fast marching method, Cambridge: Cambridge University Press, 1999

[9] V. Caselles, F. Catte, T. Coll, F. Dibos, A geometric model for active contours in image processing, Numer. Math., vol. 66, pp. 1-31, 1993 